\title{
Métrologie autour d'installations plasma-laser. Utilisation du rayonnement synchrotron
}

\author{
Ph. Troussel, C. Bobin ${ }^{1}$, C. Rémond, Ph. Stemmler et B. Villette \\ CEA-DAM lle de France, BP. 12, 91680 Bruyères-le-Châtel, France \\ ${ }^{1}$ MICRONICA Technologie et Recherches, La Ferranderie, 28120 Montigny, France
}

\begin{abstract}
Résumé : Dans le cadre du projet Simulation du CEA/DAM, l'accès expérimental aux hautes densités d'énergie et au domaine de la fusion thermonucléaire en Laboratoire se fera par le Laser Mégajoules (LMJ) dont le prototype LIL. est en cours de construction au CEA/CESTA près de Bordeaux.

Les programmes expérimentaux correspondants ont nécessité des performances élevées et le développement d'une instrumentation de premier plan. C'est notamment le cas des diagnostics plasma qui ont évolué avec le développement des lasers de puissance et celui des technologies adaptées pour étudier leur comportement. Le Service Diagnostics Expérimentaux du CEA/Bruyères-Le-Chatel développe des diagnostics et intègre la métrologie associée dans la conception de diagnostics. Nous présentons les moyens dont on dispose auprès du rayonnement synchrotron pour la métrologie des diagnostics et l'intérêt de cette métrologie pour l'étude des plasmas-laser.
\end{abstract}

\section{INTRODUCTION}

L'objectif de la métrologie est d'abord de pouvoir comparer des résultats expérimentaux avec les données des codes de simulation. Dans ce but, il faut caractériser les fonctions de transfert du point de vue spectral, spatial, efficacité... La précision de la mesure et en particulier la détermination de grandeurs absolues sont des éléments déterminants pour valider les modèles utilisés pour la physique des plasmas.

Pour «mesurer le plasma », nous utilisons des grandeurs physiques : émission X, température, densité, état d'ionisation. L'objectif est double : connaître le spectre de rayonnement $\mathrm{X}$ émis par un plasma, si possible à l'aide d'une mesure absolue, connaître la dimension et l'évolution des zones émissives.

Un diagnostic se compose généralement d'un détecteur, ou capteur, précédé d'un système optique. Ce dernier peut être aussi bien une véritable optique qu'un simple diaphragme ou un simple système dispersif souvent associé à des filtres.

\section{NOS MOYENS DE MESURE}

Le premier travail de l'étalonnage est d'abord de choisir sa source de rayonnement $\mathrm{X}$ puis le détecteur de référence. Pour le premier point, nous choisissons en priorité le rayonnement synchrotron : ce rayonnement de type «impulsionnel répétitif » est l'outil de recherche privilégié. L'anneau Super-ACO du LURE est intéressant car il délivre un spectre dont le domaine spectral est voisin de celui émis par les plasmas laser. Notre laboratoire dispose de deux montages :

- Au dessous du keV, une chambre expérimentale «GONIOXUV » ou goniomètre XUV (Figure 1) amovible utilisée par campagne derrière des lignes équipées d'un monochromateur à réseaux. 


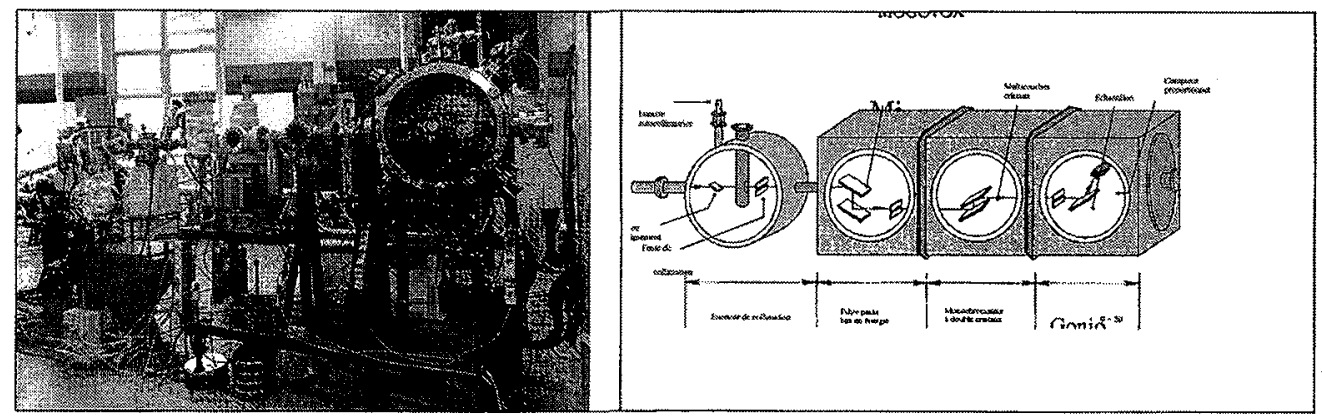

Figure 1 : Expérience GONIOXUV

Figure 2 : MOGOTOX

- En dessus d'1 keV, un monochromateur MOGOTOX [1] (Figure 2) à deux cristaux de la ligne SB3. de conception analogue à celle de GONIOXUV, la précision de la mécanique est supérieure, à cause de l'exigence imposée par l'utilisation des cristaux (angulairement $10^{-4} \circ$ et spatialement $1 \mu \mathrm{m}$ ).

Dans une expérience d'étalonnage il faut au préalable déterminer la distribution spectrale du rayonnement de la source. Dans le cas du synchrotron BESSY II au PTB ", on peut utiliser directement la source comme source primaire de référence [2]. Le flux de photons est annoncé avec une incertitude de $0,4 \%$. Il suffit ensuite de placer le capteur sur le faisceau pour en déterminer sa réponse. La source de Super-ACO n'offre pas les caractéristiques permettant une telle utilisation; c'est donc le capteur qui va servir à étalonner la source comme étalon de transfert (étalon primaire) pour réaliser les expériences comparatives avec le détecteur à étalonner (étalon secondaire). Le premier objectif a donc été de développer un (ou plusieurs) capteur(s) jouant le(s) rôle(s) d'étalon(s) primaire( $s$ ), et adaptés au milieu synchrotron.

Les principaux détecteurs susceptibles de remplir cette fonction se distinguent en fonction de leur mécanisme de fonctionnement :

- les compteurs proportionnels : d'une utilisation aisée, ils présentent les inconvénients de tous les détecteurs munis de fenêtre : fonction de transfert liée à la fenêtre, efficacité de détection limitée à basse énergie. A haute énergie, le rendement d'absorption du gaz de la chambre est insuffisant et les spectres sont distordus.

- les détecteurs semi-conducteurs en silicium compensés au Lithium Si(Li) : ils ont les mêmes inconvénients dus à la fenêtre d'entrée. Avec un refroidissement à l'azote liquide, les Si (Li) sont intéressants pour leur excellente linéarité sur trois à quatre ordres de grandeur, ils sont incontournables en spectroscopie en raison de leur bonne résolution.

- Les semi-conducteurs classiques" : il s'agit de diodes $p-n$, généralement en silicium ou en germanium. Etant donnée la souplesse d'utilisation et la grande dynamique de ces détecteurs (4 à 5 ordres de grandeur), ce sont les détecteurs que nous utilisons le plus couramment pour les mesures photométriques.

Il nous est donc apparu intéressant de développer un détecteur dans le but d'améliorer la précision $(<1 \%)$ qui est actuellement au mieux entre 3 et $10 \%$, d'étendre le domaine spectral ( $20 \mathrm{eV}-20 \mathrm{keV})$, mais aussi d'augmenter la sensibilité. Les bolomètres, basés sur la mesure de l'énergie déposée sous forme de chaleur dans un monocristal par une particule ou photon et supposés être quasi-absolu (efficacité proche de $100 \%$ ), peuvent répondre à ces spécifications. Développés initialement pour l'infrarouge, ces détecteurs peuvent aujourd'hui être optimisés pour la métrologie des rayonnements $\mathrm{X}$. Le domaine qui nous intéresse tout particulièrement est le domaine des rayons $\mathrm{X}(50 \mathrm{eV}-5 \mathrm{keV})$. Dans notre cas, le fonctionnement de ce type de détecteur nécessite un système cryogénique, capable d'être régulé à une température proche de $1,4^{\circ} \mathrm{K}$. Notre photomètre a été développé en

\footnotetext{
'Physikalisch-Technische Bundesanstalt de l'anneau de stockage de Bessy à Berlin, Allemagne.
} 
collaboration avec l'équipe de Spectrométrie Thermique de l'IAS dirigée par N. Coron, qui a mis à disposition le détecteur bolométrique composite. Il dispose d'un double étalonnage in situ : électrique avec l'implantation d'une source électrique stable dans le temps grâce à une résistance de chauffage en Carbone et photonique intégrant une fibre optique dirigée vers la face avant du cristal. La faisabilité de ce détecteur a été démontrée en l'utilisant pour la détection individuelle d'une particule alpha avec une source ${ }^{241}$ Am parfaitement quantifiée $(\mathrm{E} \approx 5,5 \mathrm{MeV})$; il a donc été possible de déterminer un seuil de détection de l'ordre du $\operatorname{MeV}\left(\approx 1,610^{-13} \mathrm{~J}\right)$.

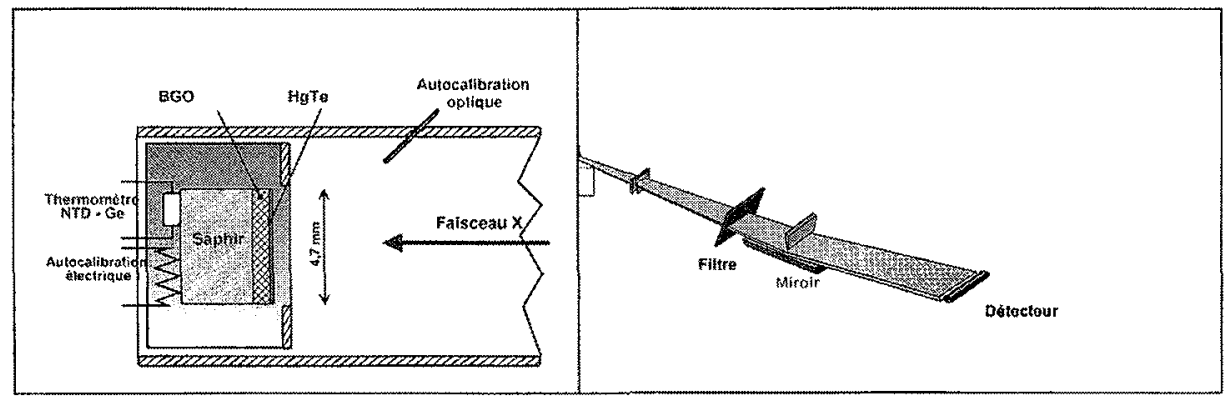

Figure 3 : Schéma du photomètre

Figure 4 : Schéma du diagnostic Demixart

\section{METROLOGIE LIANT SYNCHROTRON ET PLASMA : LE DIAGNOSTIC DEMIXART}

Jusqu'aux années 1990, différents diagnostics ont été développés pour analyser spectralement et temporellement l'émission $X$, comme par exemple en utilisant la Spectrométrie dite large bande. Dans ce cas, on mesure la quantité d'énergie émise par échantillonnage, c'est-à-dire dans un ensemble de tranches d'énergie $\Delta \mathrm{E}$ centrées sur une énergie $\mathrm{E}$. Le spectre est ensuite reconstitué avec un pouvoir de résolution de quelques unités. La conception et la réalisation par $\mathrm{B}$. Villette en 1997 [3], d'un nouveau spectromètre nommé DEMIXART (Dispositif d'Etude de L'Emission X Avec Résolution Temporelle) nous ont fait avancer notablement dans cette spectrométrie. Le diagnostic est composé de 15 voies indépendantes réparties sur une gamme spectrale s'étalant de 0,1 à $5 \mathrm{keV}$. En dessous du $\mathrm{keV}$, la sélection de la bande spectrale de chacune des voies est assurée par l'utilisation conjointe d'un miroir sous incidence rasante éliminant la composante d' $X$ durs et d'un filtre mince (résolution spectrale $\delta \mathrm{E} / \mathrm{E}=0,1$ ). Pour cela, le miroir est placé sous un angle d'incidence tel que l'énergie de coupure associée se situe juste après le seuil de photoabsorption du filtre. Audessus du keV, les cinq voies sont en direct (sans miroir).

Ce diagnostic a été entièrement étalonné auprès du rayonnement synchrotron. A titre d'exemple, la figure 5 présente la réponse spectrale d'une photocathode en aluminium établie à partir des mesures sur les lignes SA23 et SA22. On a constaté la présence inattendue du seuil de photoabsorption du Carbone déposé au cours de la fabrication ainsi qu'une dispersion importante de sensibilité (20\%) entre tous les détecteurs coaxiaux liée à cette pollution. L'incertitude sur la mesure de chaque détecteur est de $10 \%$ et de $\pm 20 \%$ sur l'ensemble des fonctions de transfert des voies du diagnostic en associant les erreurs faites sur la réflectivité des miroirs, la transmission des filtres et les réponses des diodes. Le détecteur de référence était une photodiode XUV de silicium. Les voies possèdent en outre une résolution temporelle de l'ordre de 100 ps grâce aux caractéristiques d'un détecteur de rayonnement $\mathrm{X}$ à structure coaxiale . Demixart a été un diagnostic couramment utilisé sur le laser Phebus. Prenons l'exemple d'une cible de cuivre irradiée avec un éclairement de $10^{15} \mathrm{~W} / \mathrm{cm}^{2}$. Les spectres d'émission mesurés en intégrant sur le temps mettent en évidence la présence de raies pour 
des énergies situées au-delà de $1 \mathrm{keV}$. Elles correspondent à des transitions de la couche $\mathrm{L}$ (Figure 6). Les carrés correspondent aux voies de mesure du diagnostic corrigées des fonctions de transfert. La courbe en trait continu correspond au spectre obtenu avec un spectromètre à réseau en transmission ayant une meilleure résolution spectrale.

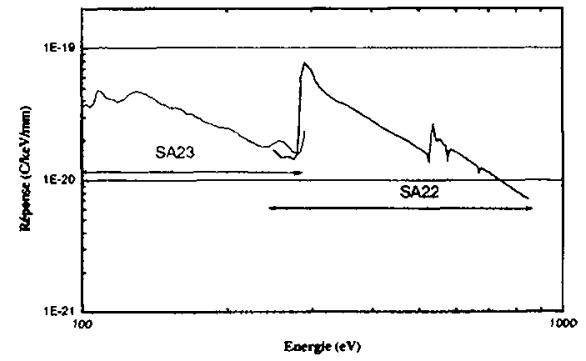

Figure 5 : Réponse spectrale de photocathode en aluminium.

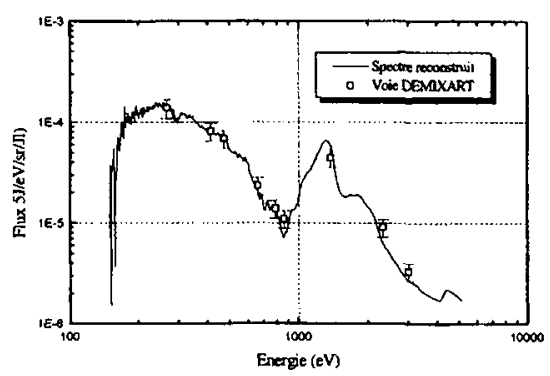

Figure 6: Distributions spectrales mesurées Jors d'un tir unique sur cible de Cuivre.

La validation des modèles d'interaction laser matière demande de réaliser des mesures absolues avec des incertitudes de plus en plus faibles, la phase de métrologie fine précédant l'expérimentation nécessitera toujours le rayonnement synchrotron et une mise à disposition de lignes fortement instrumentées. Le bolomètre refroidi devrait s'imposer comme le détecteur d'avenir étant donné sa précision inférieure au pourcent [4].

\section{Références}

1. L. Beck, P. Stemmler,et al, « Detector calibration at CEA/Bruyères-le-châtel », Nuclear Instruments and Methods in Physics Research A 369 401-406 (1996)

2. D. Arnold, G. Ulm, « Electron storage ring BESSY as a source of calculable spectral photon flux in the X-ray region », Rev. Sci. Instrum. $63 \mathrm{~N}^{\circ} 1$, (Janvier 1992)

3. J. L. Bourgade, B. Villette et al « DMX : An absolutely calibrated time resolved broad soft $x$-ray spectrometer designed for MJ class laser produced plasmas, RSI à paraître fin 2000

4. G. Ulm et al «The PTB radiometry laboratory at the Bessy II electron storage ring», Proc. SPIE, 3444, pp. 610-621, (1998) 